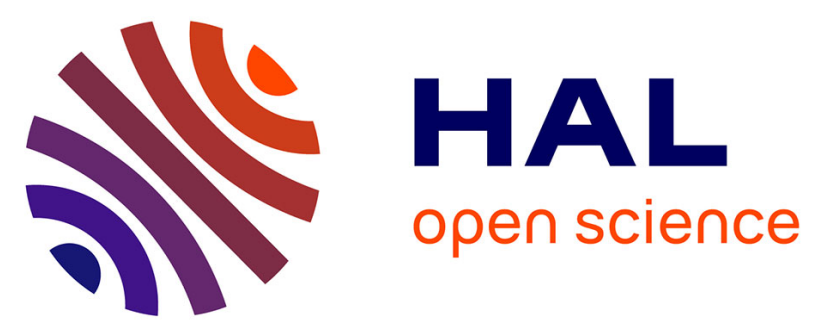

\title{
An agent-based model for high-density urban redevelopment under varied market and planning contexts
}

\author{
Simone Zarpelon Leao, Benoit Gaudou, Christopher J. Pettit
}

\section{- To cite this version:}

Simone Zarpelon Leao, Benoit Gaudou, Christopher J. Pettit. An agent-based model for high-density urban redevelopment under varied market and planning contexts. Reed, Richard; Pettit, Chris. Real Estate and GIS: The Application of Mapping Technologies, Châpitre 7, Routledge - Taylor \& Francis, pp.116-139, 2018, 978-1315642789. 10.1201/9781315642789-7 . hal-03033989

\section{HAL Id: hal-03033989 \\ https://hal.science/hal-03033989}

Submitted on 5 Jan 2021

HAL is a multi-disciplinary open access archive for the deposit and dissemination of scientific research documents, whether they are published or not. The documents may come from teaching and research institutions in France or abroad, or from public or private research centers.
L'archive ouverte pluridisciplinaire HAL, est destinée au dépôt et à la diffusion de documents scientifiques de niveau recherche, publiés ou non, émanant des établissements d'enseignement et de recherche français ou étrangers, des laboratoires publics ou privés. 


\title{
Chapter 7
}

\section{An agent-based model for high-density urban redevelopment under varied market and planning contexts}

\author{
Simone Z Leao ${ }^{1}$, Benoit Gaudou ${ }^{2}$ and Chris Pettit ${ }^{1}$ \\ 1 City Futures Research Centre, University of New South Wales, Sydney, Australia \\ 2 Institut de Recherche en Informatique de Toulouse, Universite Toulouse 1 Capitole, France
}

\begin{abstract}
Compact city policies implemented through mixed-use higher density urban redevelopment are reshaping cities worldwide. There is lack, however, of analytical tools integrating information on the existing built form, land market and planning controls that could provide an assessment of how much redevelopment an area can promote over the future, how economically feasible this development is, and if it would meet the demand for housing. Without this, planning will remain responding to individual pressures from redevelopment pushing the boundaries to higher densities without a clear understanding of its consequences at larger scales. This study describes an agent-based model to fulfil this task. It innovates from previous modelling initiatives because it employs actual geographical data, operates at parcel level, provides a 3D dynamic visualisation, and allows the assessment of scenarios. The test of the model to a real case study demonstrated the strength of the model in handling geographic data easily, providing insights of the combined effects of land market and planning framework on the urban redevelopment process, and its ability to be linked to urban design processes to assess their actual delivery potential based on economic feasibility. Current advances in high performance computing and the increasing availability of urban big data raise optimistic horizons for further development of realistic agent based models to assist better understanding, planning and management of urban property development over the future.
\end{abstract}

Key words: Urban redevelopment; High density; Agent-based model; 3d visualisation; GIS data; Economic feasibility.

\section{Introduction}

Compact city policies implemented through mixed-use higher density urban renewal and infill development are reshaping cities worldwide (Randolph 2006; OECD 2012; UN Habitat 2013). In the past, most of the urban growth occurred at the fringes of cities through expansion and sprawl, and also in some large parcels often centrally located made available for conversion into housing after relocation of manufacturing and industrial activities to the outskirts of urban areas or offshore into cheaper labour markets. As these central spaces start to become scarce, and as governments become more concerned with the additional infrastructure costs associated with fringe dwelling construction, there is increasing attention directed towards areas of existing housing to provide additional dwellings for a growing population (Pinnegar et al., 2015).

Figure 1 illustrates the context faced by many urban planners in relation to high density urban redevelopment. Figure 1.a shows that theoretically, all the space up to the maximum height permitted by the planning framework (controls) can potentially be redeveloped into new buildings. In reality, however, only part of that space is 'eligible' for redevelopment every year (Figure 1.b). The eligibility depends on the age of the existing building; whether it is for sale, and if it is economically feasible to redevelop the site (assuming a minimum profit and the existing planning framework). Redevelopment projects can be proposed within the current planning maximum height; or above that (Figure 1.c). In NSW/Australia, the latter type of proposal, if refused by the local urban planning agency, can be taken by the developer to the State Joint Regional Planning Panel (JRPP), where 
developers and planners have the opportunity to defend their positions, and the panel dictates the final outcome.

This tension is in part caused by the different objectives and scales of assessment between urban planners and developers. While the first look at the functioning of precincts with the goal of overall quality of life of a community, developers tend to look at individual sites and the profitability of the development. Since planning also need to respond to housing future demand, to promote the revitalization of areas and allow economic growth through redevelopment, it should establish its planning framework controls (e.g. maximum height) carefully, and also have some systematic way to time future upgrades of these controls. A development proposal significantly above planning controls in an area with large availability of suitable and economically feasible sites for redevelopment within the existing planning framework will probably cause overall negative effects, by changing the character of an area, letting an individual firm capitalise most of the required supply, and creating a precedent for further development of this type before the area has matured for that high density. On the other hand, if a development significantly above planning controls is proposed in an area with scarce or no availability of suitable and economically feasible land for redevelopment, it may indicate the need for a revision of current planning controls, and potentially the increase in the maximum height permitted in some parts of a precinct. This context is very relevant for the profession of property and real estate, since the interplay on land market forces and planning regulation affect the supply and the price of properties in an area under redevelopment.

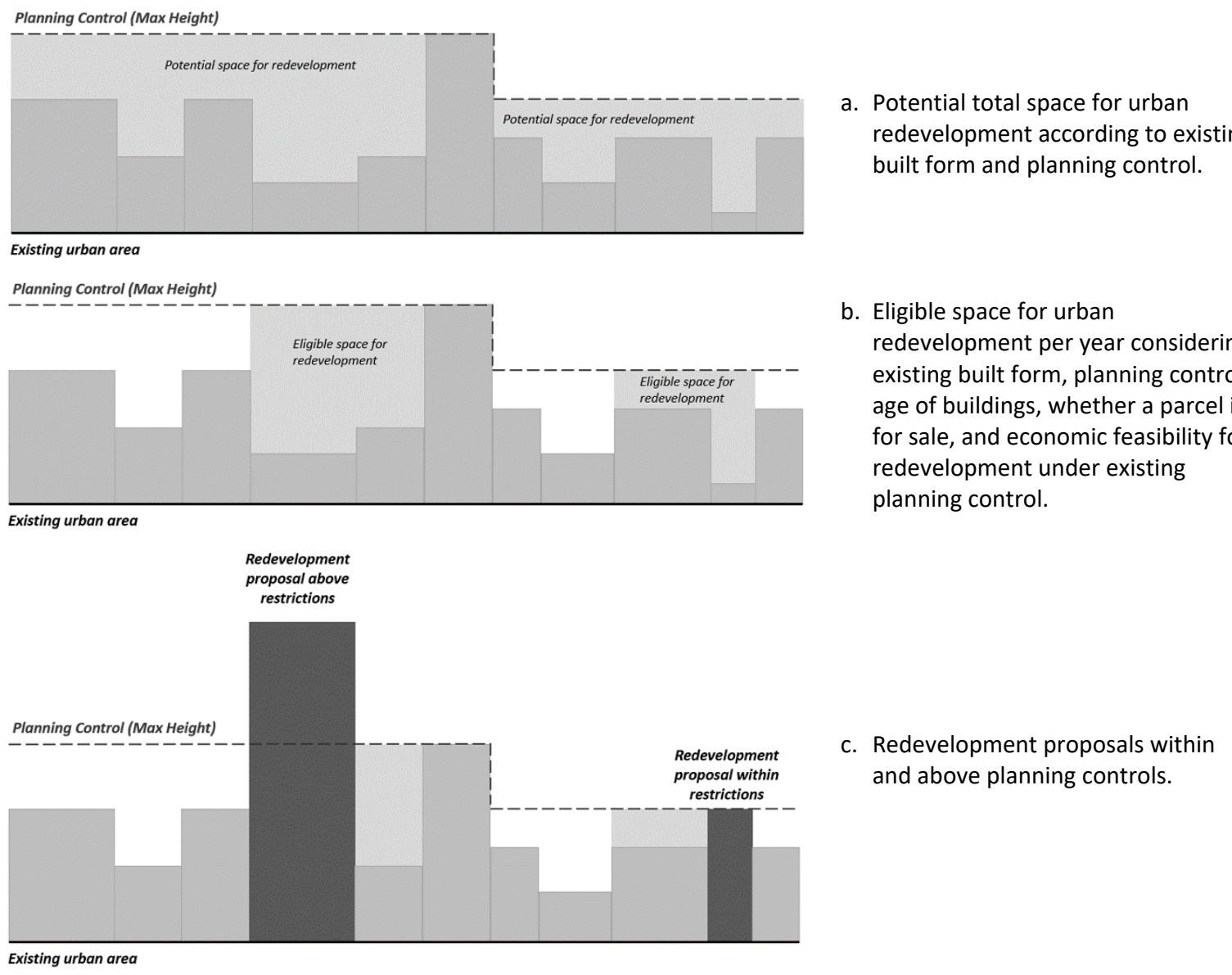

Figure 1. Potential, eligible and proposed space for urban redevelopment: hypothetical example of a precinct skyline

This chapter argues that the decision on whether approving or not proposals above planning controls, and the revision of planning control parameters, should be based on analytical processes integrating information on the existing built form, land market and planning controls; these should provide an assessment of how much redevelopment an area can promote which is economically feasible for developers, and if this potential redevelopment meet the demand for housing in the area. To the best of our knowledge, there is a lack of such type of analytical tools. And in that context, planning will remain responding to individual pressures from 
redevelopments pushing the boundaries upwards without a clear understanding of its consequences at larger scales.

\section{Background}

Some previous research brought to light relevant dimensions associated to the urban redevelopment process, and also presented some analytical methodologies to investigate urban redevelopment dynamics. Helms (2003) focused his study on factors affecting urban rehabilitation. He developed an empirical analysis of determinants of housing renovation in areas under gentrification. Although this is not the same of urban redevelopment, these two processes have in common the investment in improving the built environment and often the change to a higher density. Helm found that age, size, and number of units were significant factors associated to characteristics of the property being rehabilitated; and also indicated that zoning and planning characteristics, not included in his study, deserves further investigation. Troy et al (2015a, 2015b) investigated the economic feasibility of urban redevelopment processes at a parcel level. They developed empirical rules for assessing the economic feasibility of medium and high density urban redevelopment of multifamily properties across Greater Sydney in Australia, unravelling a process strongly driven by the market with significant influence from the planning framework and neighbourhood characteristics. Their approach included similar factors previously identified by Helms (2003), with the addition of the planning framework. A limitation, however, of this approach is that the model was originally non-spatial and static.

Since the process of urban redeveloped is spatial, Leao et al. (in press, accepted on 19 Oct 2017) progressed on the previous model, developing a version of Troy et a's model within a geographical information system (GIS) platform. This new version of the model allows users to visualise geographically different scenarios of urban redevelopment according to varied decisions of planning controls, and also variations in the market conditions. A survey with urban planners testing the model in a participatory workshop indicated the model is very useful planning support system tool.

A potential route to overcome the temporal limitation was proposed by Parker and Filatova (2008). They argue that land markets have unique features that make them appropriate for agent-based modelling: they are an heterogenous commodity traded by heterogeneous agents; spatial and agent-agent interactions can be present; and they operate in non-equilibrium dynamics. With this is mind, they developed a conceptual design of an agent based model of some aspects of urban economics, with focus on better understanding the interactions between multiple buyers and sellers and the results in land value. Later, Filatova et al. (2009) progressed on the former work and implemented and tested the conceptual framework into an agent-based model of urban land markets. Developed in Netlogo platform, the model used a hypothetical monocentric city represented as a generic grid, where various artificial buyer and seller agents negotiate land prices for residential purposes. Levy at al. (2013) developed this approach further, including developers as agents, and assessing outcomes of land markets associated to urban densification. This model also tested theoretical hypothesis of urban economics in a hypothetical and generic grid-based monocentric city. Although being rich platforms for scientists to test hypothesis of urban economics, the generic agent-based models described above have limited applicability for urban planning or real estate purposes in real urban settings. There is a lack of agent-based models of urban land markets built on actual urban geographies and current economic and planning data.

City planners and urban professionals need new tools and methodologies to gain insights into ways to deliver effective responses to the push towards greater urban densification considering the interplay between land and property markets and planning frameworks. This study describes an agent-based model to fulfil the task by assessing the economic feasibility of urban redevelopment within actual precincts at a parcel level, considering existing and potential built form, and parameters associated to the current planning framework and land and property markets, and providing a visual portray in 3D of the dynamics of buildings redevelopment. Using a real case study, the model performance was successfully evaluated in terms of its capacity to produce metrics and visualisations of potential scenarios of redevelopment.

The process being modelled in this chapter is the vertical development of urban precincts from low or medium to high or very high density; in other worlds, detached houses or old multi-storey apartment buildings being knock-down and replaced by modern high-rise buildings. This chapter presents a quite unique approach to represent urban change in an agent-based environment, in which parcels are autonomous agents; they are fixed in the bi-dimensional space, but they can change their height through redevelopment and densification. The parcels' behaviour is driven by ageing, suitability, availability, economic feasibility, planning compliance, and ultimately by replacement if a series of conditions are met. These conditions are dependent on the parcels initial conditions and some external parameters (defined by the market and urban planning). There are many agent- 
based urban models reported in the literature, but generally they are generic and hypothetical (such as Filatova et al. 2009 and Levy et al. 2013 described before), or when they are more realistic, they have buildings as background and the modelled processes are driven by mobile agents such as people or vehicles. To the best of our knowledge, this is the first attempt to model the process of urban redevelopment using an ABM approach with parcels/buildings as autonomous agents. A 3D environment has been selected as the most suitable form of visualisation of the transformations simulated by the model. Moreover, as actual data can be available for precincts of interest, and most urban planning agencies now work routinely with Geographic Information Systems (GIS), this research also decided to develop the agent-based model in a platform able to work with spatialised information.

\section{Research questions and discussion}

Within the context of property and real estate profession and the specific case study analysed, three questions are posed: (1) What insights the agent-based model or urban redevelopment is able to provide to users? (2) Can the model be used to investigate potential future scenarios based on neighbourhood design proposals? And (3) what are the main benefits and limitations of agent-based modelling for urban redevelopment research?

\section{Research methods}

Agent based modelling ( $A B M)$ is a method under the umbrella of complexity. It is built upon the principles that emergence of macro-patterns come from individualised but interconnected micro-decisions. The concept of 'micromotives and macrobehaviour' was already stated in the homonymous book published by Thomas Schelling in 1978. However, it was the advances in computing that allowed its translation into sophisticated computer models (Crooks et al 2008). In ABM, autonomous agents interact with the environment and with each other through behavioural rules. Although the rules can be simple, the varied configurations of the environment and the agents may cause non-linear feedback, and result in surprising and unexpected results (Axelrod 2005). Agents can be people, vehicles, countries, or urban parcels, as in the model presented here. Chen (2012) argues that the foundation and concepts of agent based modelling are particularly suitable in socio-related studies, especially in urban studies and design project management.

This section presents the proposed urban redevelopment ABM following the ODD protocol - Overview/Design Concepts/Details (sub-sections 4.1 to 4.3, respectively) (Grimm et al. 2006). This protocol has been widely accepted and used as the standard to describe agent-based models since its proposal, and confirmed appropriate for urban models (Polhill et al. 2008). Finally, sub-section 4.4 describes the modelling platform selected.

\subsection{Overview}

\subsubsection{Purpose}

The purpose of the model is to understand how land and property markets (purchase cost, sale revenue, profit expectation) and urban planning frameworks (buildings' maximum height and floor space ratio) affect high density urban redevelopment (economic feasibility, regulation compliance, regulation revision, and housing supply) at a parcel and precinct levels.

\subsubsection{State variables and scales}

The model comprises two hierarchical levels (from high to low): precinct, and parcel. Parcels are characterised by the state variables: area; type (built or vacant); age; whether it is a strata title; number of units/dwellings; current height; maximum height according to current planning framework. 'Strata title' allows individual ownership of part of a property (generally an apartment), combined with shared ownership in the remainder (common areas of the property, such as driveways, foyer, gardens, lifts, etc) through a legal entity called the owners corporation; it offers a legal mechanism for space to be vertically subdivided and traded, allowing individualised property rights to be applied to multi-unit housing (Troy et al 2017). According to the current NSW Strata regulation, at least $75 \%$ of the units, through their owners' votes, are required to approve the sale a whole strata building for redevelopment (http://stratalaws.nsw.gov.au/).

Since parcels can be redeveloped (old building replaced by new building, or vacant land filled with new building), its state variables (building age, height, number of units/dwellings, and whether it is a strata, etc) can change. A precinct, a higher level entity, is formed by parcels (with or without buildings) with fixed area and location. Its properties are summations and averages of the attributes of its parcels: number of parcels (total, suitable, available, compliant, redeveloped), number of dwellings/units, average building height. 
Each model run/step is equivalent to one year; and the time horizon is theoretically unlimited, but it is constrained by a 'saturation' parameter (default is $90 \mathrm{~m}$ building height for the study area, equivalent to approximately 30 floors).

The model is designed for a precinct level, with parcels as spatial units. The data used is GIS-based.

\subsubsection{Process overview and scheduling}

The parcels in the precinct can change their attribute as a result of two processes: ageing and redevelopment, being the later one driven by the developer; also, the planning framework may be revised by the urban planner according to criteria associated to land scarcity and density (Figure 2). The process of redevelopment contains a series of interlinked sub-processes: suitability, availability, amalgamation, economic feasibility, and (re)placement. The planning framework encompasses sub-processes which influence redevelopment, including planning compliance, and framework revision.
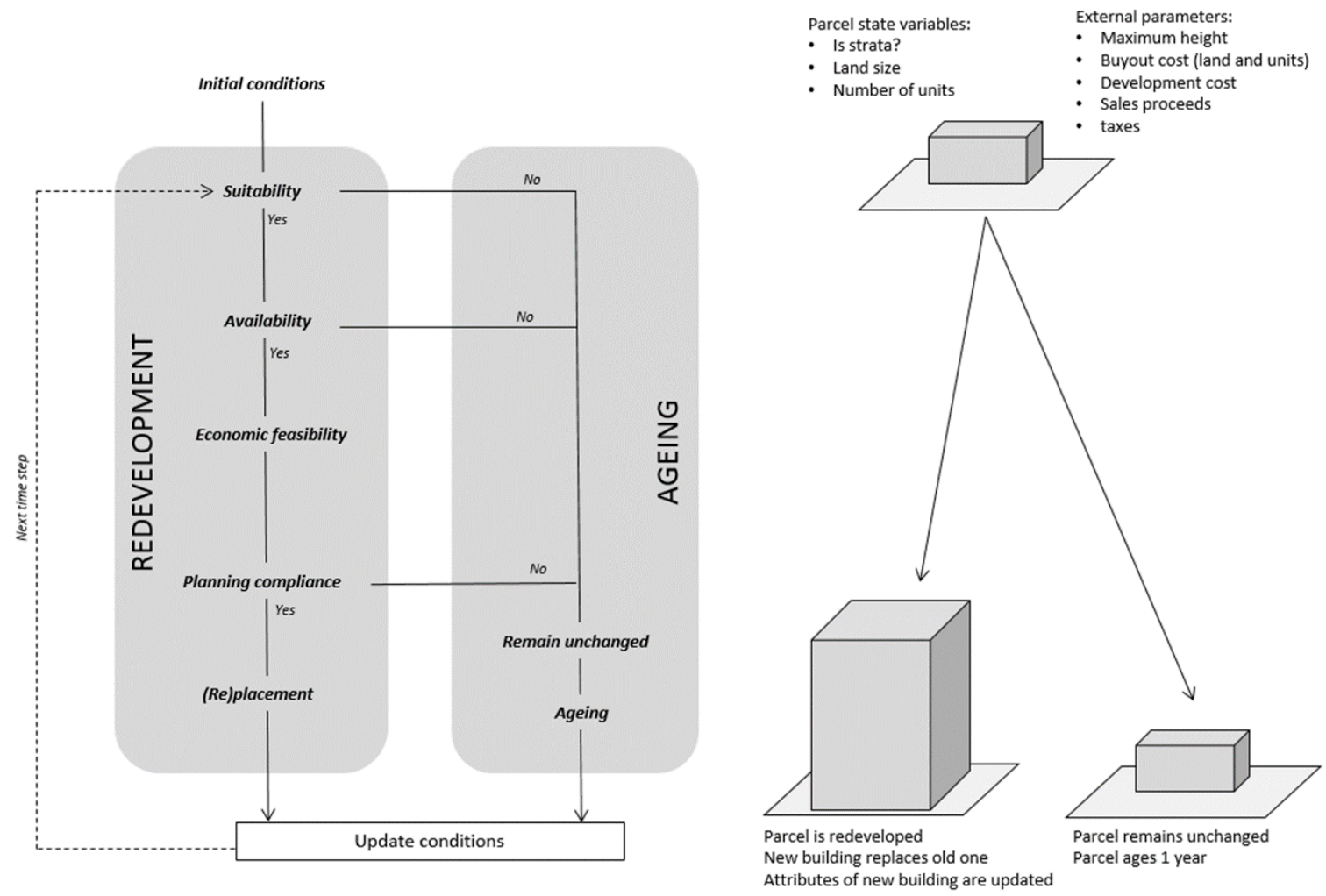

Figure 2. Simplified scheme of model processes

Ageing: The age of a building is a criterion for its suitability to be redeveloped. Only building which are at least 25 years old are suitable for redevelopment. The age of an existing building is added one unit at the end of every model step, which represents a year. Every time a building is replaced by a new one as a result of redevelopment, its age is re-set to zero, and the ageing process goes on from that at the next step.

Redevelopment: As a result of the redevelopment assessment a parcel can be redeveloped, which involves the replacement of an existing building by a new one, or 'placement' of a new building in a vacant land; or the parcel remains unchanged. The redevelopment assessment is comprised of a series of stages which run at each time step of the model. They are described below:

- Age Suitability: First, the suitability of a parcel for redevelopment is assessed based on its age; only parcels with buildings which are 25 years or older are considered 'suitable' for redevelopment. Vacant parcels are always suitable for redevelopment.

- Availability on the market: Second, the availability of parcels previously classified as suitable is assessed. This process tries to emulate the fact that although suitable, not all parcels would be available on the market for sale at a time. Based on a random number, parcels are attributed a binary state (available or not available). 
- $\quad$ Economically feasible urban form: Fourth, the model calculates the built form required for a new building to be economically feasibility, considering a minimum profit required by the developer (by default it is $20 \%$ ). This process is developed for the individual parcels previously classified as suitable and available, and also for the potential amalgamated sets of parcels. The calculation is calibrated to local land and property market conditions and construction costs at a precinct level. The new built form required for economic feasibility is described in terms of number of new dwellings/units and number of floors of the new building.

- $\quad$ Compliance to framework: Forth, the model compares the number of floors of the new building (to be economically feasible) to the maximum height permitted by the planning framework at the location (converted into number of floors). If the new height is below or equal to the maximum threshold, the building is considered 'compliant' and the replacement of the old building can be performed in the next stage; otherwise, the old building remains unchanged at this time step.

- (Re)placement of old building or vacant land: If a parcel passed positively through all the previous assessments, being suitable, available, and compliant to planning at economic feasibility height, it is listed as a candidate for redevelopment. Candidates will be selected and redeveloped within a model time step while the sum of new units provided by redevelopment minus the sum of units knocked-down is below the annual housing demand for the area. The candidate parcels list is ranked in descending order of profit to development at maximum height, so the most profitable parcels available will be given preference. The attributes of the redeveloped parcels are updated with the characteristics of the new building.

After the processes of 'ageing unchanged parcels' and 'updating the attributes and age of new buildings', a new time step of the model is run again, and so forth, until the saturation parameter is reached.

\subsection{Design concepts}

\subsubsection{Emergence}

Redevelopment dynamics emerge from parcels' behaviour, but their life cycle and behaviour are almost entirely represented by empirical rules describing, for example, suitability, economic feasibility, control compliance, and replacement. Exception is for the availability, which is random. Urban redevelopment is emergent in the sense that it is the result of decentralised decisions of autonomous 'parcels'. Collectively these decisions will result in a housing supply (overall number of residential units) and an urban high density landscape (number of floors of building by year in a 3D visualisation).

\subsubsection{Fitness}

Fitness-seeking is not modelled explicitly, but is included in the empirical rules. At an individual agent level, fitness is measured by the economic feasibility with compliance to planning framework, and agents decide to redevelop or not at a time step based on their fitness.

\subsubsection{Sensing}

For simplification at this version, parcels are assumed to be autonomous agents, aware of their state attributes, aware of land market and planning conditions, able to evaluate their economic feasibly and compliance with planning, and capable of 'redevelop' themselves if conditions are appropriate.

\subsubsection{Interactions}

The model does not model the interplay between owners of old buildings/units, developers, buyers of new units, and planners. For simplification at this first version of the model, parcels are assumed to be the only agents. The assessment they perform on themselves to decide if they redevelop or not are through functions which included information from planners and developers. Moreover, parcels are assumed to be completely independent, the fact that a parcel is for sale or have been redeveloped recently does not affect the neighbouring cells. Indeed, the parcel-agent represents the behaviour of a developer willing to pay market price for a property and develop it attending the planning controls if it can achieve at least $20 \%$ profit after sales of new units constructed. The model allows interaction with the user, through the manipulation of model parameters associated to land and property markets, planning framework, and some characteristics of new urban form.

\subsubsection{Stochasticity}


The availability of a parcel for sale at the land and property market at every single year (model step) is a random attribute for a parcel with low frequency (few parcels at a time). This means that a parcel for sale in one year may not be available at the following year, even though it has not been sold and redeveloped. Also a parcel for sale in the market, although available, may not be economically feasible for development at the planning controls at the time, and not be redeveloped for that reason. This means that even in a case study with real parcels attributes and calibrated empirical rules for land market and planning controls, each simulation will result a different outcome, due to the stochasticity of the properties availability on the market, and the fact that all the subsequent assessments for urban redevelopment are dependent of the characteristics of the parcels available. This also means that, although parcels are agents "aware" of economic and planning context (they represent the action of a developer), property sellers are not. This is considered appropriate, because not all parcel owners are necessarily potential sellers waiting for the best opportunity. Some may have long-term intentions to stay at their properties, or need to sell a property at a certain time regardless market opportunities or adverse conditions.

\subsubsection{Observation}

Observation includes a 3D visualisation of the parcels in a precinct. It starts showing current heights of buildings at time 0 of the simulation, and at each model time step, parcels redeveloped are highlighted in colour (in comparison to the ones which remain unchanged) and also extruded in scale to the height of the new building. Graphs summarise some model outcomes: (a) number of suitable and redeveloped parcels in the precinct; and (b) average building heights vs planning maximum height in the precinct.

\subsection{Details}

\subsubsection{Initialisation}

This model has been developed to work with actual data. The main dataset is a GIS layer file with polygons representing parcels, with an associated attribute table containing the state variables of each parcel. The study are has around 1,500 parcels with varied sizes, age, and density of development. The current planning framework establish maximum height varying from 3 to 10 floors, FSR from 0.5 to 3 excluding the town centre area, where FSR is not limited. Moreover, the model requires the user to input some parameters related to costs and revenues of new development. For the case study, all attributes have a default value, a suggested range for meaningful manipulation. These are: sale price of an old existing unit is AU\$ 785,000; sale price of a new redeveloped unit is $A \cup \$$ 800,000; price for vacant land is $A \cup \$ 1,125 / \mathrm{m}^{2}$; construction cost of a predominant typical typology in the area is AU\$215,000 (a 2-bedroom apartment with balcony and medium standard quality); the annual demand for new residential units is 200 units/year; the size of the new redeveloped units is $90 \mathrm{~m}^{2}$ each; site coverage is $60 \%$; and the proportion of parcels for sale is $30 \%$. Using an actual case study, the initial state variables are always the same. However, the random number used to define 'availability' on the market, will make each model run a unique thread. Creation of scenarios is possible in the model, as a result of changing state variables in the attribute table of the parcel dataset, and also, from new values for model parameters (profit margin, land coverage, buyout cost, sale proceeds, annual housing demand, etc).

\subsubsection{Input}

GIS feature data (polygon shapefile) with attribute table describing associated state variables; parameters calibrated to a study area.

\subsubsection{Sub-models}

Sub-model 1 - Suitability:

'Suitability' of a parcel is tested at the start of every time step, as buildings are ageing (increased age) or being replaced (decreased age) along the urban redevelopment process. The parcels type and age of an existing building are used as criteria for the suitability assessment, as shown in Figure 3. 


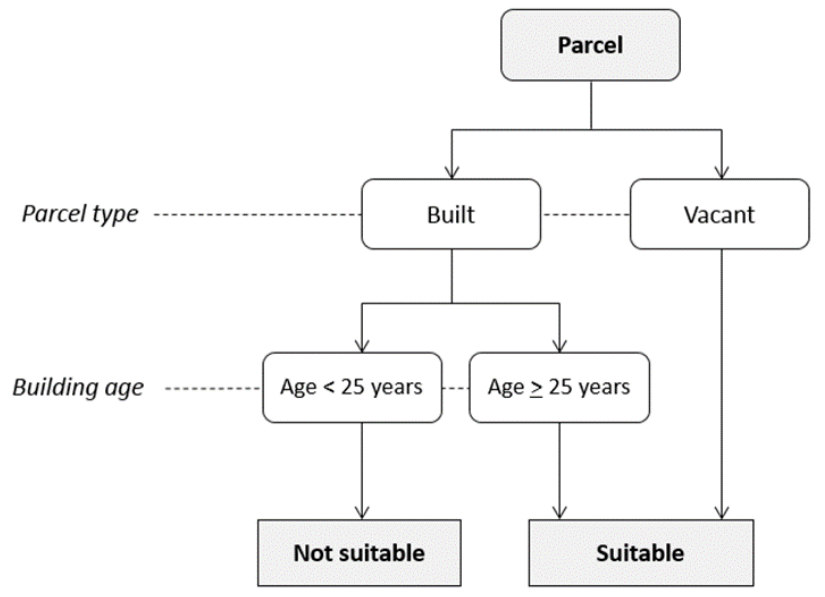

If ('parcel is built' and 'existing building age $>=25$ ' or 'parcel is vacant'),

then parcel is 'suitable';

otherwise, parcel is 'not suitable'.

Figure 3. Suitability assessment for urban redevelopment

Sub-model 2 - Availability:

'Availability' state of a parcel is tested at the beginning of every time step, after sub-model 1. Whether a parcel is 'available' is defined through a random assignment with low frequency (tendency to have less available than unavailable parcels in the market) (Figure 4).

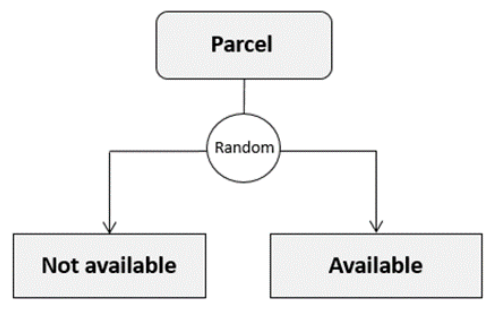

Figure 4. Availability assessment for urban redevelopment

Sub-model 3 - Potential amalgamations:

For each parcel which is 'suitable' and 'available', the model calculates the number of units and number of

Sub-model 3 - Height of economically feasible building:

For each parcel which is 'suitable' and 'available', the model calculates the number of units and number of floors a new building would need to be economically feasible to be redeveloped. The calculations evaluate all the costs involved in purchasing land (in the case of non-strata properties) or units of a building (in the case of strata properties), paying taxes, and constructing new units of a certain size; as well as all the revenues from their sales. To be economically feasible the new building must have a size able to provide the developer a profit of at least $20 \%$ (all revenues minus all costs). The sequence of calculations and the description of the variables and constants used in the model are shown in Figure 5. 


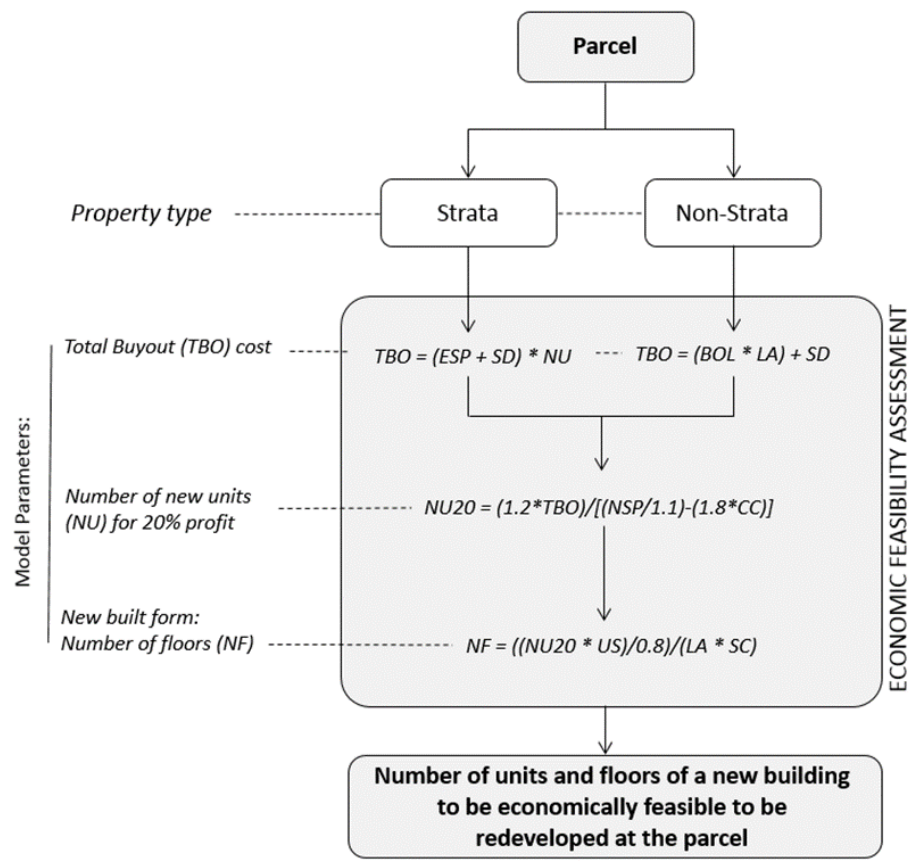

$N U=$ Number of units in a strata property (units) $L A=L a n d$ area of a non-strata property (sqm) $E S P=$ Sale price of existing strata unit (\$) $B O L=$ Sale price of non-strata property by sqm of land $(\$ / s q m)$ $S D=$ Stamp duty (tax over property purchase)

$N S P=$ Sale price of new unit $(\$)$

$C C=$ Construction cost of new unit (\$)

US $=$ Unit size (sqm)

$\mathrm{SC}=$ Site coverage $(\%)$

Constants in the formulas account for: $20 \%$ developer profit (1.2); $10 \%$ GST (1.1); $80 \%$ of builders' overhead (1.8); and $80 \%$ private area in strata titles $(0.8)$.

Figure 5. Economic feasibility assessment for urban redevelopment

Sub-model 4 - Compliance with planning controls:

For each parcel which is 'suitable' and 'available', the model checks if a new building which is economically feasible complies or not with the planning framework. This assessment is made by comparing the number of floors of the new building to the maximum number of floors allowed at the parcel site. If the height of the new building is at or below the maximum threshold, the parcels is compliant, otherwise it is not compliant. This is illustrated in Figure 6.

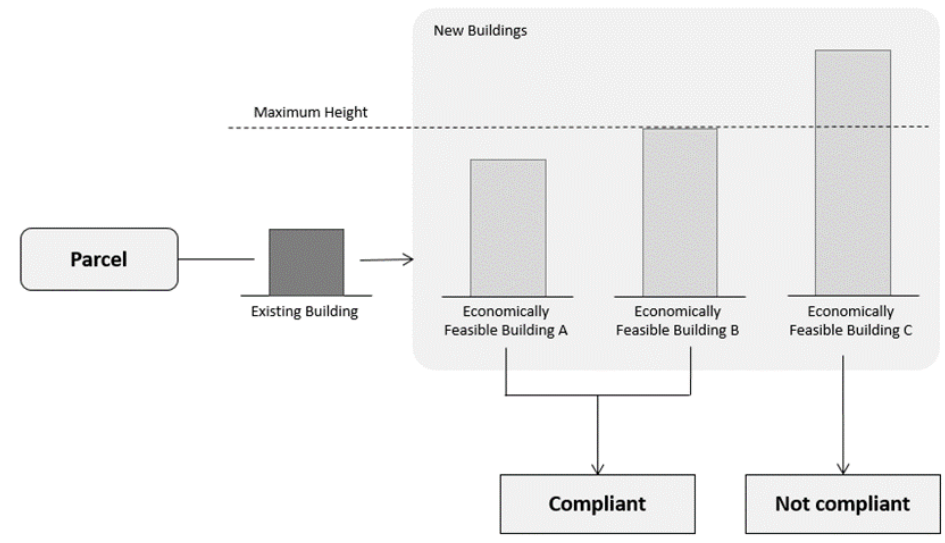

If 'New floors' <= 'Max floors'

(e.g. Buildings $A$ and $B$ ),

then parcel is 'compliant';

otherwise (e.g. Building C), parcel is 'not compliant'.

Figure 6. Planning compliance assessment for urban redevelopment

Sub-model 5 - Ageing:

If a built parcel is 'not suitable', or 'not available', or 'not compliant', it remains unchanged and ages one year at the end of every time step:

Parcel age (built) $=$ Parcel age (built) +1

Sub-model 6 - Building (re)placement:

Redevelopment involves the replacement of old building by new building or placement of a new building on a vacant land. If a parcel is 'compliant', it will be listed as candidate for redevelopment and all candidate parcels will be ranked in descending order of profitability of redevelopment at maximum height. Parcels will be redeveloped at a model time step until the housing supplied by redevelopment minus the sum of units knocked down is equal or higher than the annual demand of housing. The annual housing demand is an input provided 
by the user, and the supply is the accumulated number of units in the new redeveloped building at the minimum profitability. This process is illustrated in Figure 7. At the end of the time step, the state variables for the 'redeveloped' parcel are updated according to the characteristics of the new building defined in sub-model 3 calculations (number of units, number of floors, strata title) and age $=0$. For simplification at this first version, it is assumed the new building is immediately built.

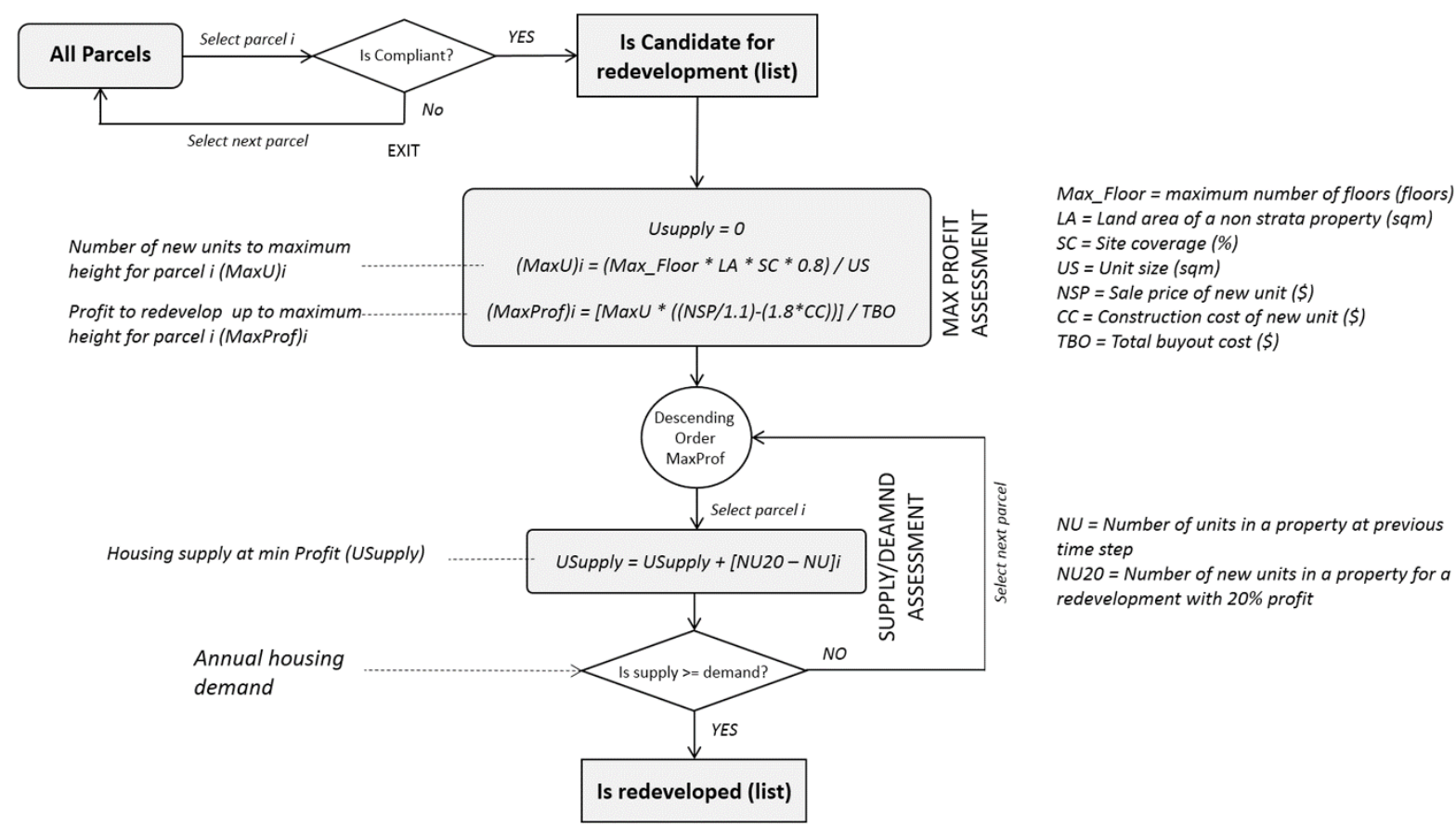

Figure 7. Selecting and redeveloping parcels

\subsection{Modelling platform}

The model has been developed using GAMA (www.gama-platform.org). This choice was based on: (a) the capability of GAMA in working with GIS data and instantiating agents from geographical features (Taillandier et al 2010); (b) its good performance with 3D visualisation of GIS data (Grignard et al 2013); and (c) and for being a free, open source and well documented platform.

\section{Results and analysis}

To test the model performance, a case study has been selected and four experiments are developed: (a) simulation with past data and comparison with actual redevelopments occurred in the study area (validation); (b) simulation with present context to get insights into future redevelopments (simulation); (c) simulation for testing the economic feasibility of an actual design proposal for the study area; and (d) simulation of a simple automated trigger for revising planning framework.

\subsection{Case study: location and parametrisation}

Kensington-Kingsford Town Centres corridor, in the South-East region of Sydney, Australia, is used as a case study in this paper to test the use of the proposed agent based model for urban feasibility assessment of urban redevelopment at parcel level.

This is considered a rich case study due to two reasons. First, the planning agency of this region has been approached by several developers with requests for construction at building heights above thresholds established by the existing planning framework. These are expected pressures for urban growth and redevelopment in the area due to its favourable location, connectivity, and high standard of services. Second, an urban design competition was launched in 2016 for this area, and the winner design proposed a significant reconfiguration for the area, without any explicit consideration for its economic feasibility (http://yoursayrandwick.com.au/k2k).

Figure 8 presents the model parameters input window, filled with calibrated values as default for the study area to assess the economically feasibility of urban redevelopment in 2016. 


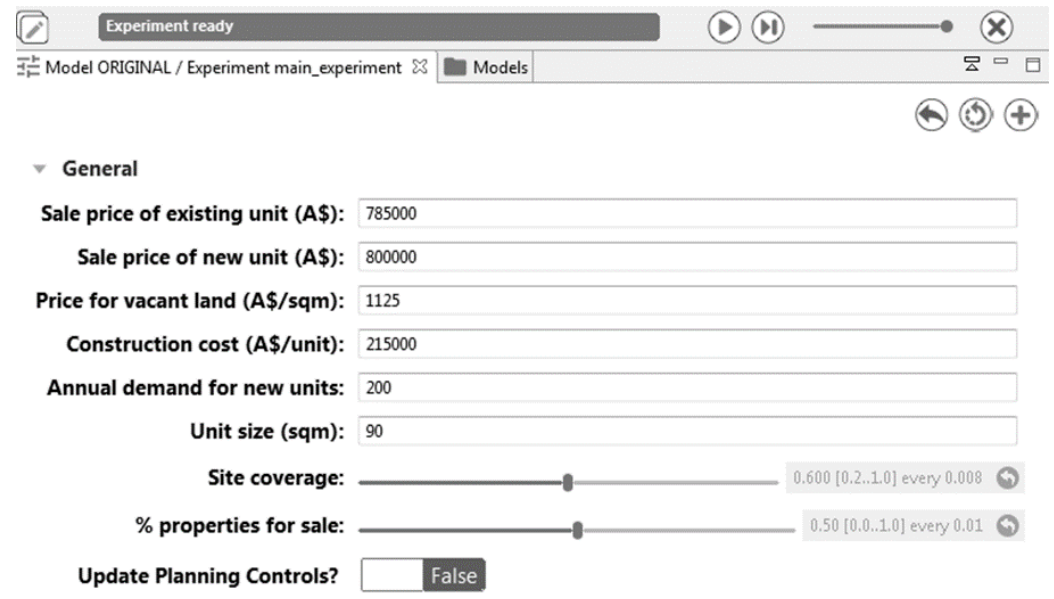

Figure 8. Model parameters for the study area

\subsection{Validation}

Data on nine new buildings that have been redeveloped on 30 parcels in the study area from 2009 to 2016 has been used to validate the model rationale and calculations. Figure 9.a locates those redevelopments in the study area and illustrates an urban redevelopment process in 2013, in which two parcels with single floor commercial units (Figure 9.b) were amalgamated and a seven-floor high rise building was built as replacement with 42 new residential units (Figure 9.c). It is tested here if the model would identify those areas as potential redevelopment sites.

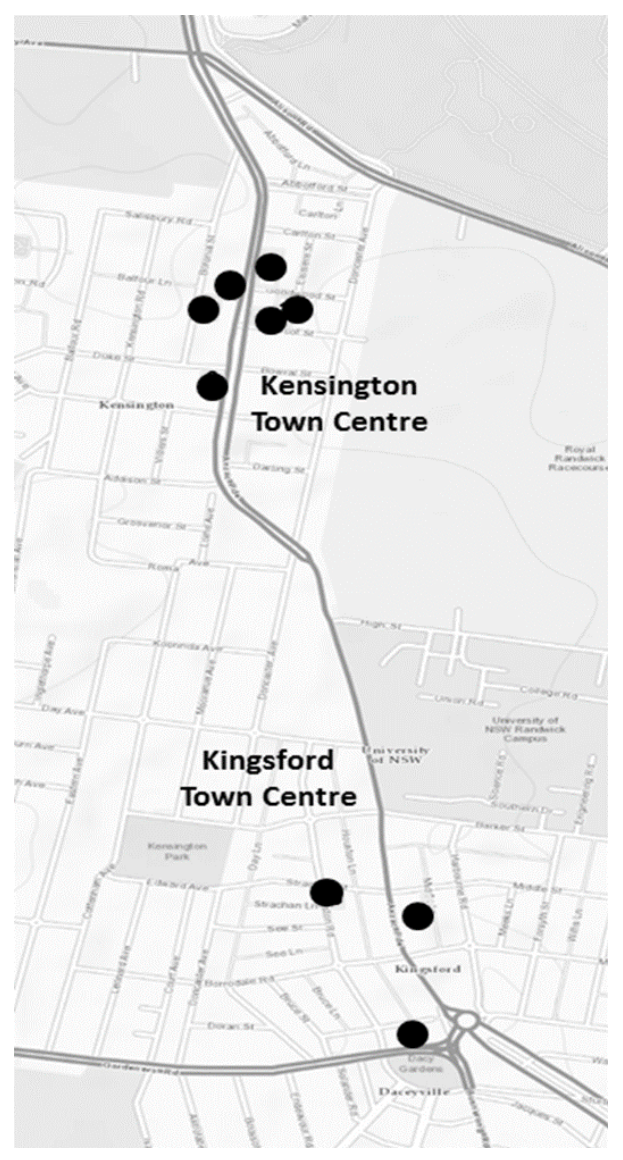

a. Actual redevelopments in the study area 2009-2016

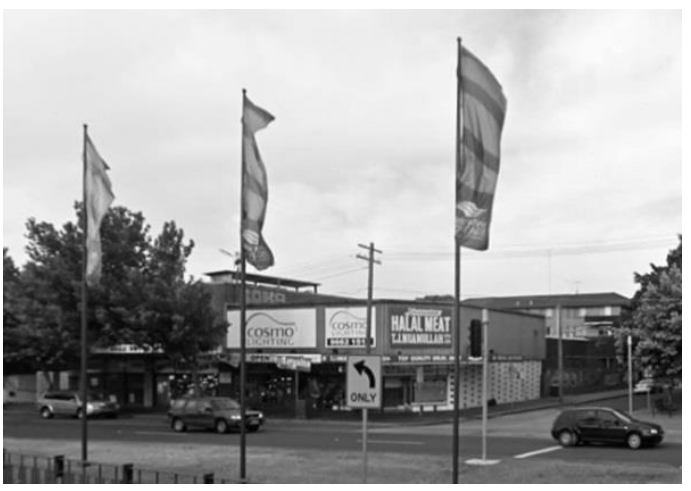

b. Before: 2 parcels with a single floor commercial unit each.

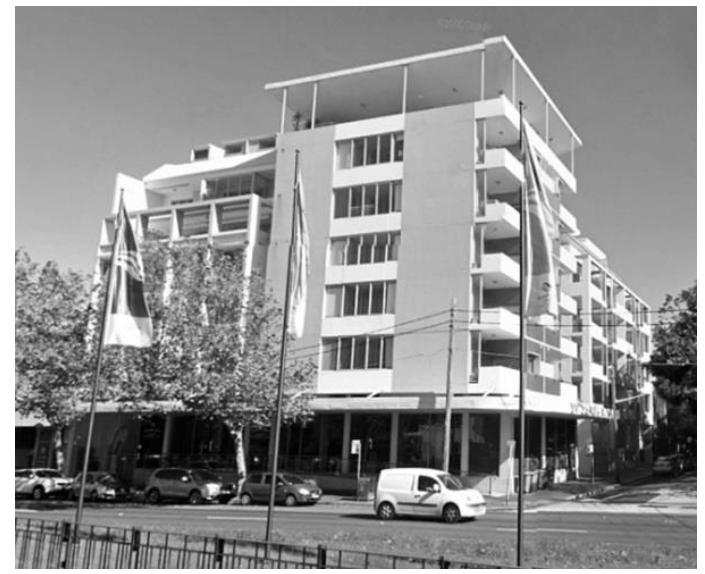

c. After: amalgamated parcels with a 7-floor building (42 dwellings), built from 2011 to 2013

Figure 9. Study area and exemplar urban redevelopment 
The Urban Redevelopment ABM was run for the context of 2009 (parcels subdivision and existing built form before redevelopments) assuming that all parcels would be available in the market, and then the results were compared to the actual redevelopments occurred in the area.

It was found that all the parcels redeveloped in the study area were also virtually redeveloped by the ABM for multiple model runs. They were all considered suitable, economically feasible and compliant with the planning framework. Also, the model showed that those parcels produced the same profit for developers than the average of all suitable \& compliant parcels in the area (42\% profit if parcels were developed up to the maximum height).

However, the model could not replicate the actual timing and order of the redevelopments occurred in the study area. This was expected, since the entry of a property in the market at every time step of the ABM is a random variable. Multiple runs of the model for the same period generate different outcomes at a parcel level, but similar outcomes when aggregated to the neighbourhood level. Therefore, the results of the model should not be taken as an accurate 'forecast' of urban redevelopment at a parcel level, but an insight on potential redevelopment and densification processes.

\subsection{Simulations}

Simulations are developed for four scenarios: (1) business as usual; (2) an urban design proposal; (3) the urban design proposal with increased planning control; and (4) automated planning control revision. These are described below.

\subsubsection{Business as usual simulation}

Based on the conditions in the study area for the year 2016, the simulations here use the model to answer the following questions: (1) How much of the area would be redeveloped if land/property market and planning control parameters remain as current? (2) Would the area reach the full densification potential as stated by the planning controls (maximum height)?

Figure 10 illustrates the results of a simulation. Multiple runs of the model resulted in comparable results. It indicates that most of the redevelopment would occur in the Kensington area (parcels in dark grey have been redeveloped, while light grey parcels remain unchanged, Figure 10a). This shows that Kingsford, in order to become economically feasible to be redeveloped, would need higher allowance for building heights, floor space ratio, or higher sales price for properties, or a combination of those. Indeed, the simulation indicates that in around 25 years there are no more eligible parcels for redevelopment (Figure 10b). Although the planning controls would allow further development, the lack of economic feasibility blocks redevelopment before reaching the full potential of the area. This indicates that a revision of planning controls for the Kingsford area may be required.

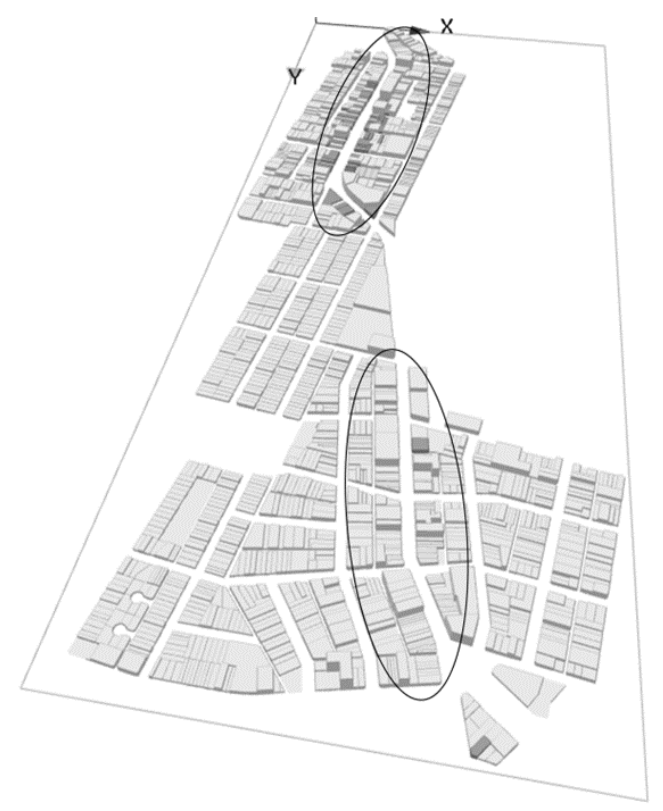

a.3D visualisation of redeveloped parcels

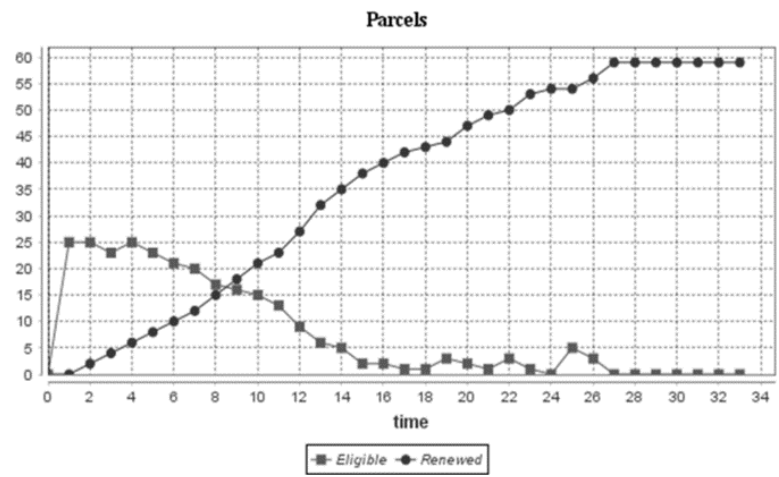

b. Eligible and redeveloped parcels annually 
Figure 10. ABM for urban redevelopment for the 'Business as usual' scenario

\subsubsection{Simulation of a winning urban design proposal}

The local government responsible for the study area launched an international urban design competition in 2016 to stimulate a future vision for the Kensignton-Kingsford Corridor region (https://www.youtube.com/watch?v=aOk7Aa-njAA). The winning design proposed significant changes to the local urban landscape and some changes to the current planning controls. These changes included the resurfacing of a creek and the creation of an extensive green-open corridor along this creek (black circle in Figure 11a). The reduction of the parcel sizes to accommodate the new green open space would be compensated by an increase in the building heights along the green corridor, which is an area currently occupied by low density housing. Increased building heights was also envisioned for the main road corridor (Anzac parade) linking the two town centres (Kensington and Kingsford, black circles in Figure 10). The design proposals for the competition had to respond to overall goals of the local government in terms of the neighbourhood image, character, economic development, service provision and quality of life. The economic feasibility of the proposals was not assessed as part of the competition process. This however is an essential component if any design proposal aims to be delivered.

The simulations here are based on the 'desired future' proposed by the winner of the urban design competition in the study area. The new parcel sizes, types, and maximum planning controls were input in the ABM. Moreover, since the design proposal did not specify a FSR, the maximum currently parameter used in the town centres was adopted in the green corridor, which is FSR 3:1. All areas not affected by the design proposal maintained their current characteristics. Land and property market parameters were considered the same as in the business as usual model.

The model is used to answer the following questions: (1) Is the urban design proposal economically feasible to be implemented? (2) How much of the area would be redeveloped in the conditions stated by the urban design proposal?

Figure 11 illustrates the results of a simulation. Multiple runs of the model resulted in similar results. When compared to business as usual, it indicates that the proposal would significantly increase urban redevelopment in the area. It would be only after 65 years that eligible parcels would no longer be available in the area. The increased redevelopments are mostly from the new area zoned as high density along the green corridor (dark grey parcels), however it is noticeable that significant part of the parcels in this new zone is not economically feasible to be redeveloped (light grey parcels). This suggests that the design proposal would not be fully development due to lack of economic feasibility, and that a revision of planning controls may be necessary to promote further redevelopment and densification of the area, as preconised in the design proposal.

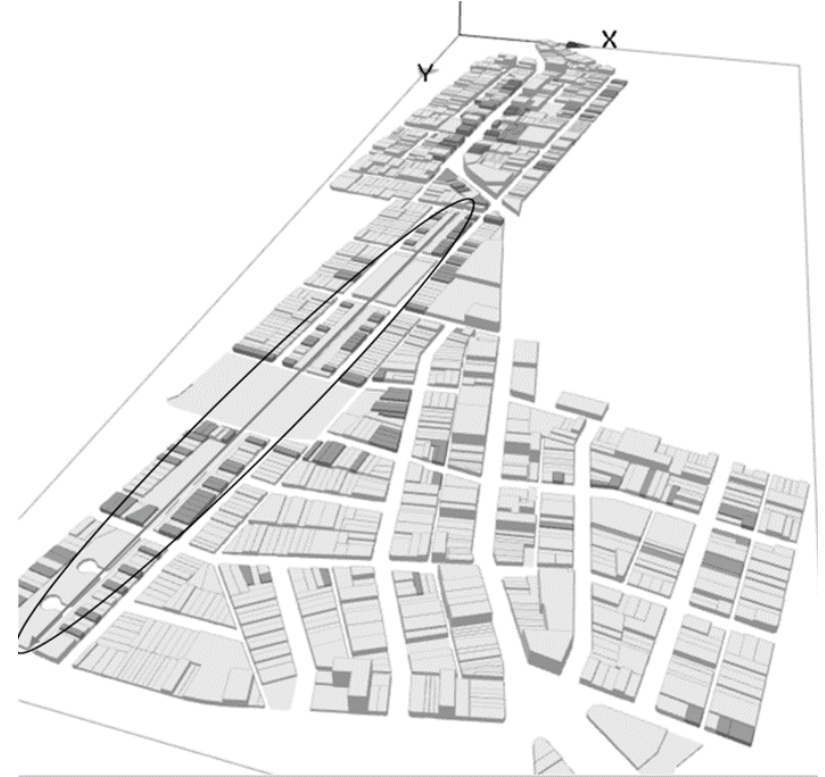

a.3D visualisation of redeveloped parcels

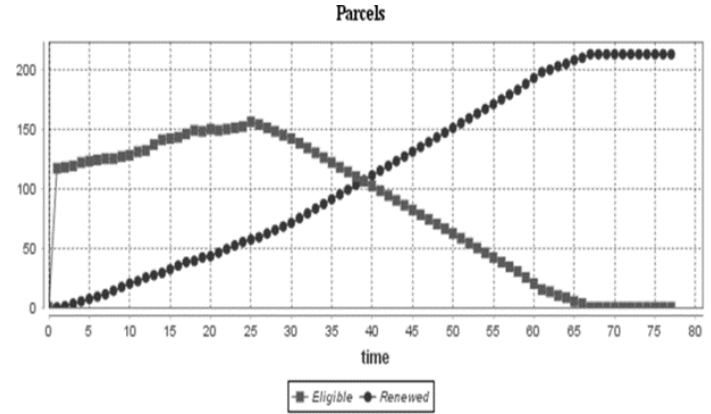

b. Eligible and redeveloped parcels annually

Figure 11. ABM for urban redevelopment for the 'Urban Design Proposal' scenario 


\subsubsection{Simulation of a winning urban design proposal with increased FSR}

Based on the results of the previous simulations with the winning urban design proposal, the effect of increasing FSR from 3:1 to 5:1 on the urban redevelopment process in the study area is tested here. The simulations use the model to answer the following questions: (1) How much of the area would be redeveloped in the FSR is increased to 5:1 in high density zones of the urban design proposal?

Figure 12 illustrates the results of a simulation. When compared to the previous simulation (5.3.2), it indicates that when FSR is increased, significant more growth occurs along the new development area, as aimed by the proposed design. This suggests the usefulness of the model to test design proposals in terms of their actual potential for implementation based on economic feasibility, which is in most cities driven by the market. The simulation results indicated that it is only from an FSR of 5:1 that the design proposal turns mostly feasible.

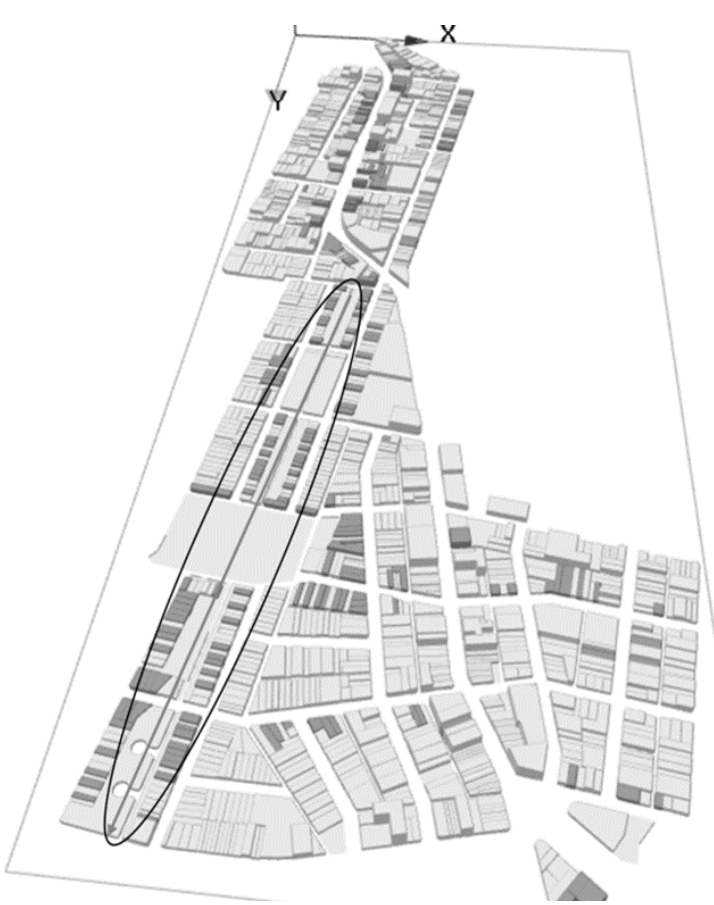

a.3D visualisation of redeveloped parcels

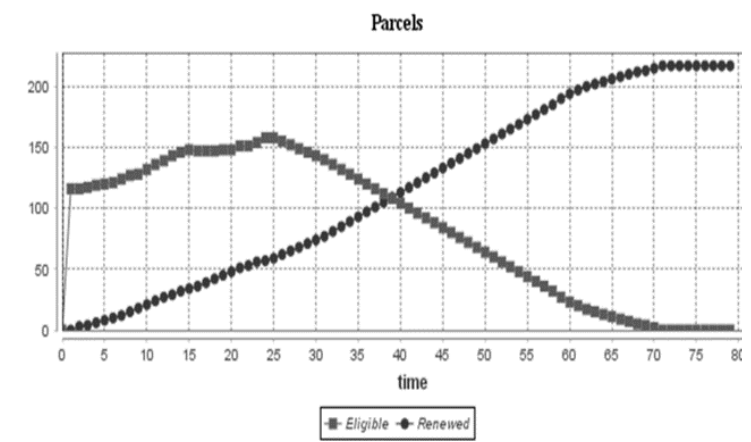

b. Eligible and redeveloped parcels annually

Figure 12. ABM for urban redevelopment for the 'Urban Design Proposal + increased FSR' scenario

\subsubsection{Automated planning control revision}

By default, the model runs progressively and at some point, when there are not more eligible parcels, the redevelopment process stops. However, the model also allows users to set an automated planning control revision process. This is done by selecting "TRUE" for the 'Update Planning Controls?' in the initial input window of the model. When this is set, the model will update automatically the maximum building height when the eligibility is low. Eligibility is considered low when potential new dwellings in eligible parcels are $20 \%$ or less of the annual demand for new dwellings; and if this occurs, existing maximum heights in the area are increased in increments of $25 \%$ (i.e. a building with maximum height equivalent to 4 floors will be increased to 5 floors; and one with 8 floors will be increased to 10 floors). This set-up makes the system continuously increase maximum height in order to avoid low eligibility. Although this is not a realistic representation of the planning controls' revision process, it assists in the visualisation of potential results of a market driven process, in which planning controls would follow land market pressures.

Figure 13 illustrates the results of a simulation, in which dark grey parcels have been redeveloped. The 'serrated' shape of the eligible parcels' graph indicate the timing of maximum height changes, and the consequent increase in parcels availability at the new planning control parameter. This occurs because with the new higher maximum height, some parcels become economically feasibility with the additional units that can be built and sold. Currently, the model runs continually until a saturation height of 120 metres is achieved. 


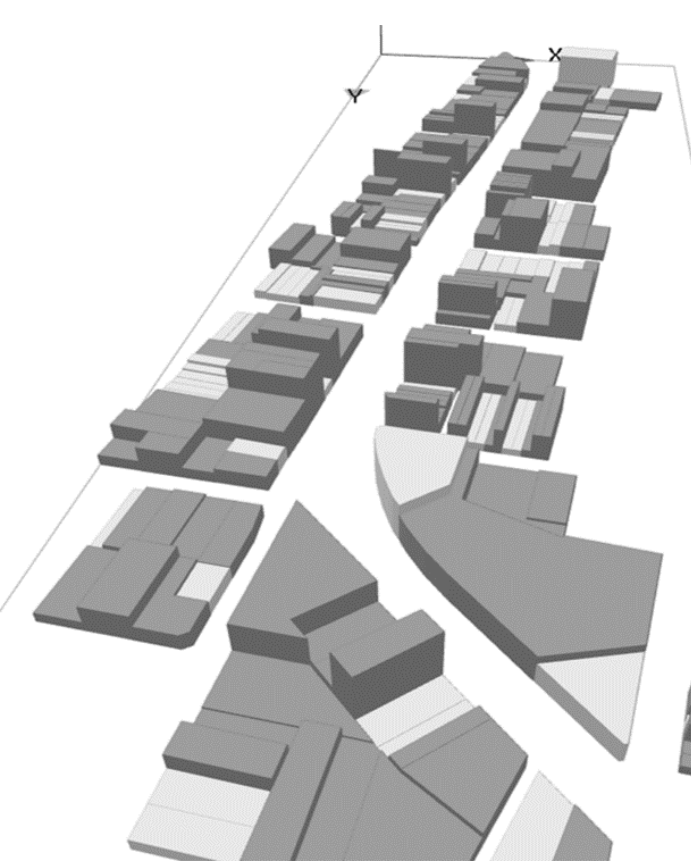

a.3D visualisation of redeveloped parcels

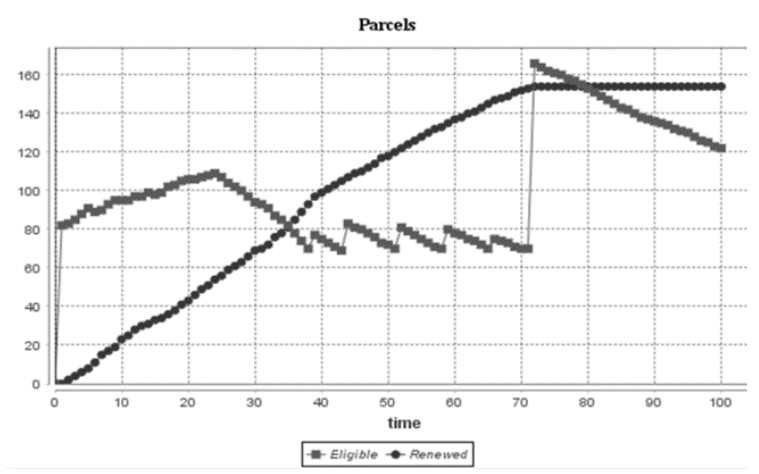

b. Eligible and redeveloped parcels annually

Figure 13. ABM for urban redevelopment with automated maximum height revision

\section{Conclusions}

This chapter presented the first version of an agent based model to assess and visualise the process of urban redevelopment of an area over time considering land market conditions and the planning framework context. One significant innovation of the proposed model is the fact that it works with actual geographies and disaggregated data at a parcel level, with a realistic neighbourhood visualisation. All the agent based models currently reported in the literature dealing with the economics of urban redevelopment use artificial and simplified cities represented by generic grids. Despite being useful to test theoretical hypothesis, these generic and simplified models have very limited application in supporting planning and decision making of areas under pressure for renewal.

The test of the model to a real case study demonstrated the strength of the model in (1) handling geographic data easily; (2) providing some meaningful insights of the combined effects of land market and planning framework on the urban redevelopment process; and (3) the potential of the model to be linked to urban design processes in order to assess their actual delivery potential based on economic feasibility.

In its first version, the model has also limitations. Currently the parcel is an agent which is aware of the developer's goals and also aware of the restrictions imposed by the urban planner at its location. The parcel reacts to these drivers. the intention here was to simplify as much as possible the variables involved in the system, still keeping the core complexity. It is aligned with the understanding that planning regulations and market conditions are the main factors driving redevelopment in consolidated urban centres, and that their effects can be summarised at a parcel level. Further development could try to model the interaction of human agents involved in the urban redevelopment process, such as property owners, real estate agents, developers, property buyers, investors, renters, Banks/home-loan, etc. These additional agents bring new goals and variables to the system. Another potential improvement is the capacity of the parcels to interact with each other, such as in an amalgamation process where the aggregation of two or more parcels may affect their capacity to be redeveloped. Finally, the tests of the model reported here included a small neighbourhood, and its application to a large scale urban area will bring new challenges. One challenge is related to the performance of the platform in terms of computing time and visualisation to handle large scale contexts, particularly if more agents and interactions are added to the model. The second challenge is associated to the data requirements. We believe that advances in high performance computation and the increasing availability of urban big data raise optimistic horizons for further development of realistic agents based models to assist better understanding, planning and management of urban property development over the future. 
Acknowledgements: The authors would like to thank Dr Laurence Troy and Prof Bill Randolph, UNSW/Australia, for sharing the empirical rules for urban redevelopment used in this research based on their previously published research and the City of Randwick Council for providing data for the case study.

\section{REFERENCES}

Axelrod R, 2005. Advancing the Art of Simulation in the Social Sciences, Chapter, In Rennard J-P (Ed.), Handbook of Research on Nature Inspired Computing for Economy and Management, Hersey, PA: Idea Group.

Chen L (2012) Agent-based modelling in urban and architectural research: a brief literature review. Frontiers of Architectural Research, 1 (2): 166-177.

Crooks A, Castle C and Batty M, 2008. Key challenges in agent-based modelling for geo-spatial simulation, Computers, Environment and urban Systems, Vol 32 (6): 417-430.

Filatova T, Parker D, van der Veen A (2009) Agent-based urban land markets: agent's pricing behaviour, land prices and urban land use change, Journal of Artificial Societies and Social Simulation, 12 (1) 3. http://jasss.soc.surrey.ac.uk/12/1/3.html

Grimm V, Berger U, Bastiansen F, Eliassen S, ginot V, Giske J, Goss-Custard J, Grand T, Heinz S K, Huse G, Huth A, Jepsen J U, Jorgensen C, Mooij W M, Muller B, Pe'er G, Piou C, Railsback S F, Robbins A M, Robbins M M, Rossmanith E, Ruger N, Strand E, Souissi S, Stillman R A, Vao R, Visser U, and DeAngelis D L (2006) A satandard protocol for describing individual-based and agent-based models. Ecological Modelling, 198 (1-2): 115-126.

Grignard A, Taillandier P, Gaudou B, Vo D A, Huynh N Q and Drogoul A (2013) GAMA 1.6: Advancing the Art of Complex Agent-Based Modeling and Simulation, International Conference on Principles and Practice of Multi-Agent Systems, PRIMA 2013: PRIMA 2013: Principles and Practice of Multi-Agent Systems pp 117-131.

Helms A C (2003) Understanding gentrification: an empirical analysis of the determinants of urban housing renovation, Journal of Urban Economics, 54: 474-498.

Leao S Z, Troy L, Lieske S N, Randolph B, Pettit C (in press, accepted on 19 Oct 2017) A GIS based planning support system for assessing financial feasibility of urban redevelopment, GeoJournal.

Levy S, Martens K, van der Heijden R and Filatova T (2013) Negotiated heights: an agent-based model of density in residential patterns. Proceedings of the 2013 CUPUM, Computers in Urban Planning and Urban Management, July 2-5 2013, Utrecht, The Netherlands.

OECD (2012) Compact City Policies: A comparative assessment. Paris: OECD

Parker D C and Filatova T (2008) A conceptual design for a bilateral agent-based land market with heterogeneous economic agents. Computer, Environment and Urban Systems, 32 (6): 454-463.

Pinnegar, S, Randolph, B \& Freestone, R. (2015) Incremental urbanism: characteristics and implications of residential redevelopment through owner-driven demolition and rebuilding, Town Planning Review, 86 (3): 279-301.

Polhill J G, Parker D, Brown D, Grimm V (2008) Using the ODD Protocol for describing three agent-based social simulation models of land use change. Journal of Artificial Societies and Social Simulation, 11 (203): ??

Randolph B. (2006) Delivering the compact city in Australia: current trends and future implications, Urban Policy and research, 24 (4): 473-490.

Schelling T C, 1978. Micromotives and macrobehavior. Cambridge, MA: Harvard University Press.

Taillandier P, Vo D A, Amouroux E and Drogoul (2010) GAMA: A Simulation Platform That Integrates Geographical Information Data, Agent-Based Modeling and Multi-scale Control, International Conference on Principles and Practice of Multi-Agent Systems, PRIMA 2010: Principles and Practice of Multi-Agent Systems pp 242-258

Troy L, Easthope H, Randolph B, and Pinnegar S. (2015a) Renewing the compact city: interim report. City Futures Research Centre, UNSW, Sydney, Australia, June 2015.

Troy L, Easthope H, Randolph B and Pinnegar S (2017) 'It depends what you mean by the term rights': strata termination and housing rights, Housing Studies, 32 (1): 1-16. 
Troy L, Randolph B, Pinnegar S, and Easthope H. (2015b) Planning the end of the compact city?, In proceedings of the State of Australian Cities Conference 2015, 9-11 December 2015, Gold Coast, QLD, Australia.

UN Habitat (2013) Planning and Design for Sustainable Urban Mobility: Global Report on Human Settlements. New York: United Nations. 